\title{
Research on the Spatial Effect of Urban Construction on Heat Island Effect in Shanghai Based on Remote Sensing Data
}

Author 1 Shuo Zhang,Shanghai Tongji Urban Planning\&Design Institutional Co.,Ltd., China

\begin{abstract}
Since the twentieth Century,global urbanization process is constantly accelerating, while the landscape pattern dominated by vegetation was gradually replaced by the landscape pattern dominated by artificial buildings. The change of climate and temperature caused by the expansion of urban construction land and the population aggregation, has aroused widespread concern. Under the background of national new type urbanization,improving the urban environment is the inevitable path to the new type urbanization.

Urban heat island effect is the most prominent feature of human activities impact on temperature, and it has many effects on environment, economy, society, health and so on. Among the existing researches related to urban heat island effect, the influence of urbanization on it has not been paid enough attention. Therefore, the research on the effect of urban construction on the heat island effect is incompletable, and the method is limited to the quantitative analysis as the spatial analysis is insufficient. Based on the existing research, while under the background of urban planning major,taking Shanghai as a typical case, this paper concentrates on the urban heat island effect under the influence of Shanghai urban construction. First of all,this paper takes the Landsat-7 ETM+ remote sensing image data of Shanghai in 01/08/2000,28/07/2010 and 28/07/2016 as the basic research data.ENVI software is used to retrieve land surface temperature on the thermal infrared band.Then this paper divides the heat island effect level,analyses the spatiotemporal distribution of land surface temperature in different years in Shanghai, and summarizes the evolution characteristics of urban heat island effect, analyzing the spatial impact of urban construction related factors such as urban scale, spatial structure and land use.

Analysis shows that city construction land expansion will aggravate the urban heat island effect. The increment of built construction in the city, the size of the resident population, as well as the population density have significant correlation with urban heat island effect,but the spatial distribution of population density and spatial distribution of heat island effect has no significant correlation.In various types of landuse, residential,industrial and mining,warehousing, commercial services, and transportation land can significantly aggravate the heat island effect, land types like green land, water area and farmland, etc can produce urban cold island effect in some individual area.At the same time, the location, area and shape of the green space and water area have an effect on the distance of reducing the heat island effect.
\end{abstract}

\section{Keywords}

Urban heat island effect, Spatial effect, Urban construction, Remote sensing data 


\section{Research Background}

Shanghai is one of the areas with the fastest urbanization development in China. The expansion of urban construction area and the agglomeration of population to the central area lead to the expansion of the urban heat island effect area year by year. In the 1960s$1970 \mathrm{~s}$, the area of the heat island was limited to $100 \mathrm{~km}^{2}$ in the central area of Shanghai.In the 1980s, it expanded to more than $400 \mathrm{~km}^{2}$. In the late 1990s, it expanded to suburban areas with an area exceeding $800 \mathrm{~km}^{2}$. The existence of heat island effect makes the Central District of Shanghai have higher extreme maximum temperature and longer duration of high temperature than the suburbs.

Urban heat island effect is an important environmental problem in a city. Urban planning and construction have an essential impact on it. The results show that urbanization contributes about $20 \%$ to $30 \%$ of the urban surface temperature warming in the past 50 years. Therefore, the impact of urbanization on urban heat island effect should be paid enough attention. It is necessary to study urban heat island effect from urban planning, urban spatial structure, urban land use and other aspects. This paper will study the impact of urbanization on urban heat island effect from the scale, structure, land use and other aspects, so as to provide new ideas for urban planning and sustainable construction.

\section{Research Data Sources}

\subsection{Remote Sensing Satellite Image Data}

The remote sensing satellite data in this paper are Landsat-7 ETM+image data, which are derived from the Geospatial Data Cloud Platform (http://www.gscloud.cn) of the Computer Network Information Center of the Chinese Academy of Sciences. In this paper, the image data of Landsat-7 ETM+in Shanghai from August 1, 2000, July 28, 2010 and July 28, 2016 are selected. The image of 2000 is Landsat-7 SCL-on satellite digital product (1999-2003), and the image of 2010 and 2016 is Landsat-7 SCL-off satellite digital product (after 2003). Detailed information is shown in Table 1.

Table 1 Landsat-7 ETM+Remote Sensing Satellite Data Source Specific Information

\begin{tabular}{|c|c|c|c|c|c|}
\hline \multirow{2}{*}{ Year } & Data Identification & $\begin{array}{c}\text { Strip } \\
\text { Number }\end{array}$ & $\begin{array}{c}\text { Line } \\
\text { Number }\end{array}$ & Data Time & $\begin{array}{c}\text { Average Cloud } \\
\text { Cover (\%) }\end{array}$ \\
\hline \multirow{2}{*}{2000} & LE71180382000214SGS00 & 118 & 38 & \multirow{2}{*}{$2000 / 08 / 01$} & 0.34 \\
\cline { 2 - 4 } \cline { 3 - 4 } 2010 & LE71180392000214SGS00 & 118 & 39 & & 2.44 \\
\cline { 2 - 4 } & LE71180382010209EDC00 & 118 & 38 & \multirow{2}{*}{$2010 / 07 / 28$} & 3.13 \\
\hline \multirow{2}{*}{2016} & LE71180382010209EDC00 & 118 & 39 & & 2.46 \\
\cline { 2 - 4 } & LE71180382016210EDC01 & 118 & 38 & \multirow{2}{*}{$2016 / 07 / 28$} & 27.19 \\
\hline
\end{tabular}

\subsection{Ground Meteorological Observation Data}

In this paper, the surface temperature data of 10 meteorological stations (Xujiahui Station, Pudong Station, Minhang Station, Baoshan Station, Jiading Station, Jinshan Station, Songjiang Station, Qingpu Station, Fengxian Station and Chongming Station) in central and suburban areas of Shanghai from 1985 to 2016 are analyzed. The data are from the temperature records of Shanghai Meteorological Bureau and Shanghai Weather Network (http://www.soweather.com). 


\subsection{Data on Economic and Social Development}

The data of economic and social development mainly include the scale and density of Shanghai's permanent population, the total area of built buildings and other indicators. The data come from Shanghai Statistical Yearbook (1984-2016), Shanghai Population Census (the Fifth Census in 2000 and the Sixth Census in 2010), and statistical yearbooks of all districts.

\section{Evolution Characteristics of Urban Heat Island Effect in Shanghai based on Remote Sensing Inversion}

\subsection{Remote Sensing Inversion of Surface Temperature in Shanghai}

Landsat-7 ETM + remote sensing satellite image is used to retrieve the surface temperature of Shanghai. Based on the available remote sensing data sources and related data in Shanghai, using ENVI5.2 software platform, using JM\&S single channel algorithm and Tan Zhihao single window algorithm to retrieve the surface temperature in Shanghai, the spatial distribution maps of surface thermal environment in different years in the study area were obtained. In order to analyze the spatial distribution pattern of surface temperature in Shanghai more intuitively, the Mean-standard Deviation Method is used to divide the surface heat field. Based on the study of Chen Songlin, the image of surface temperature in Shanghai is divided into six levels, i.e., mu (mean), $+0.5 \mathrm{std},+1 \mathrm{std}$ (standard deviation), with strong heat island area (33.1 C-45.4 C) and sub-strong heat island area, respectively. The spatial distribution maps of the heat island effect in Shanghai are obtained from the heat island area (30.6-33.1 C), the heat island area (28.1-30.6 C), the weak heat island area (25.6$28.1 \mathrm{C})$, the cool island area (23.2-25.6 C) and the cold island area (0.0-23.2 C).(Figure 1,2,3) 


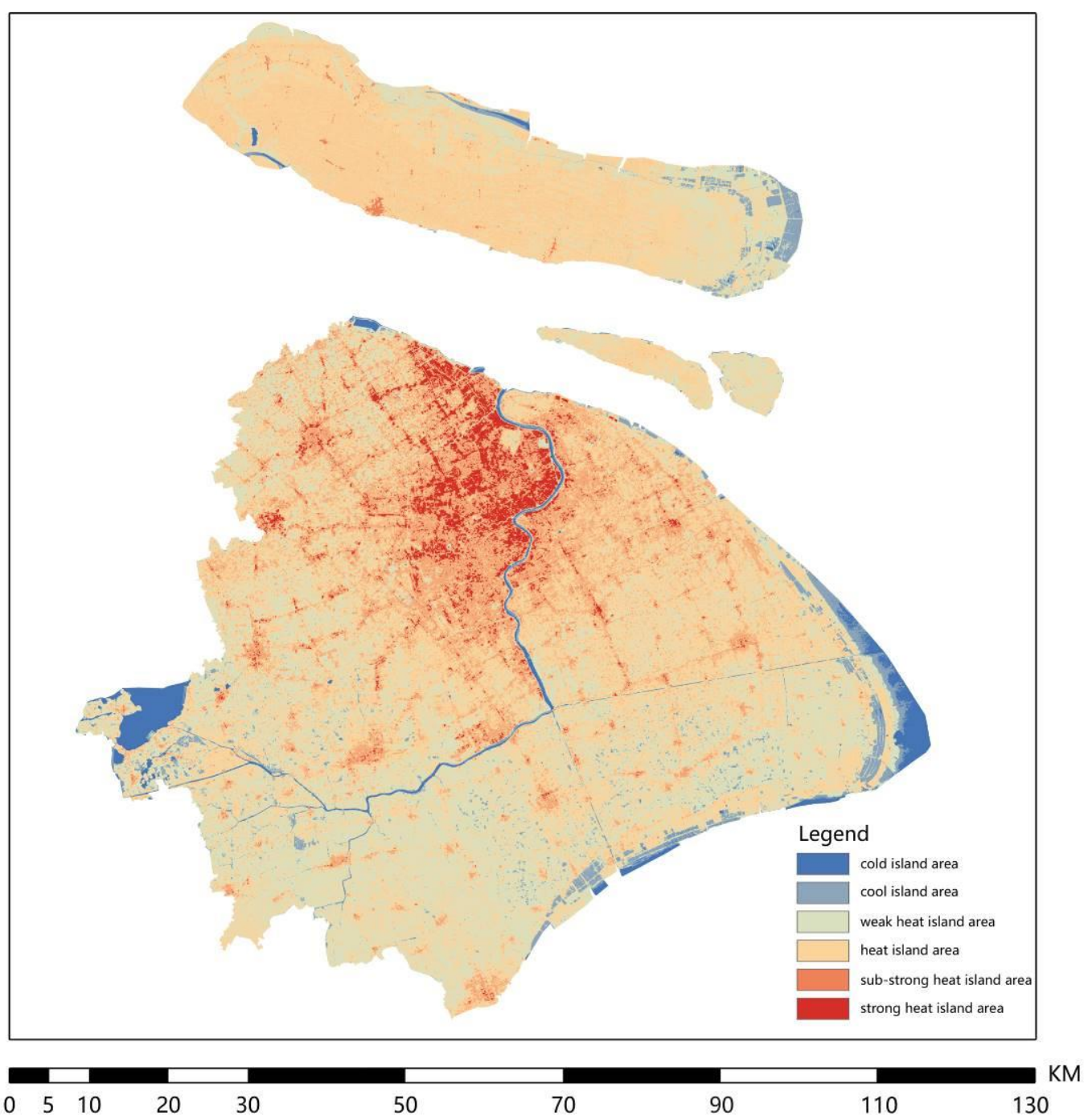

Figure 1 Spatial Distribution Map of Heat Island Effect in Shanghai on August 1, 2000 


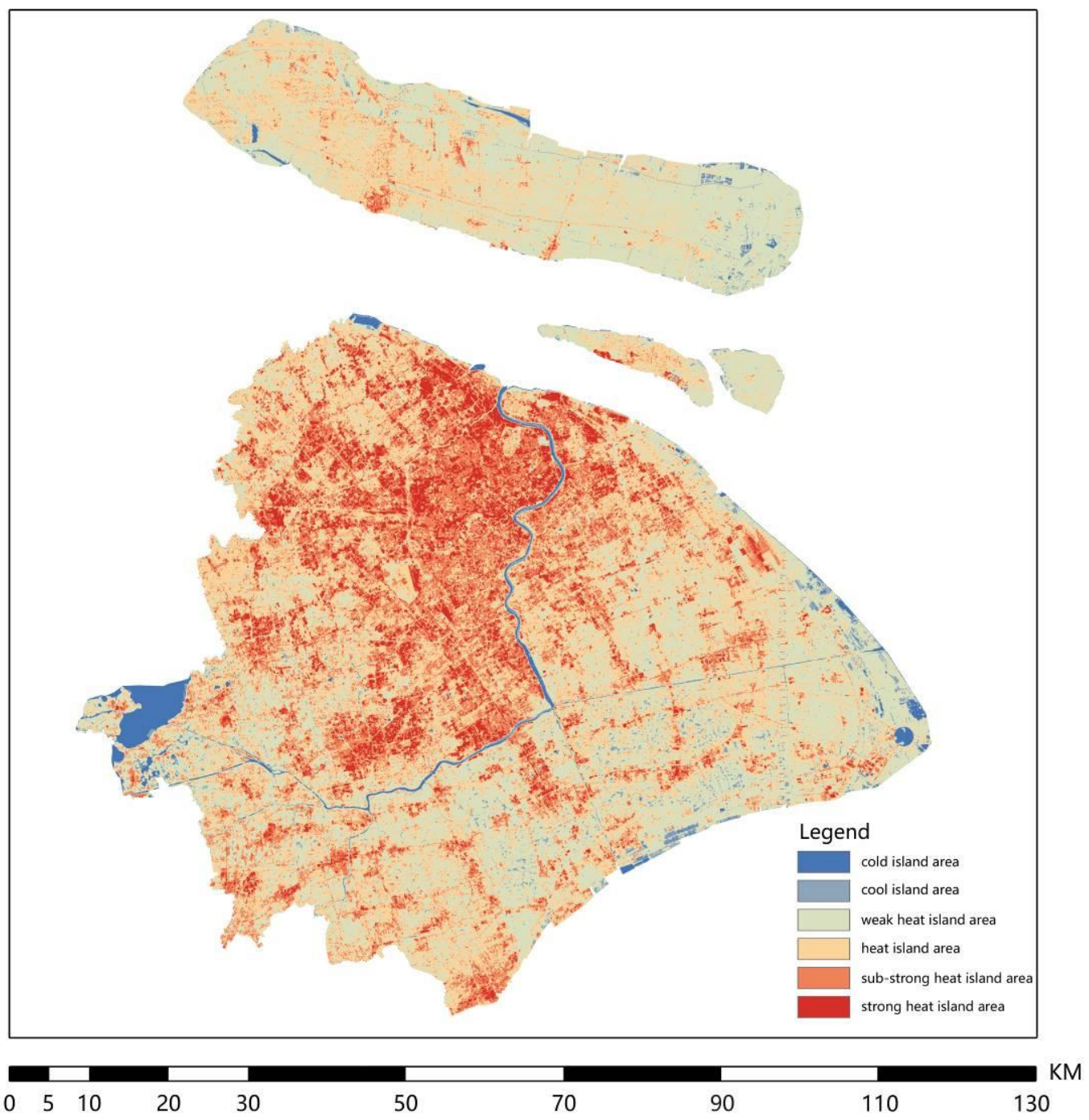

Figure 2 Spatial Distribution Map of Heat Island Effect in Shanghai on July 28, 2010 


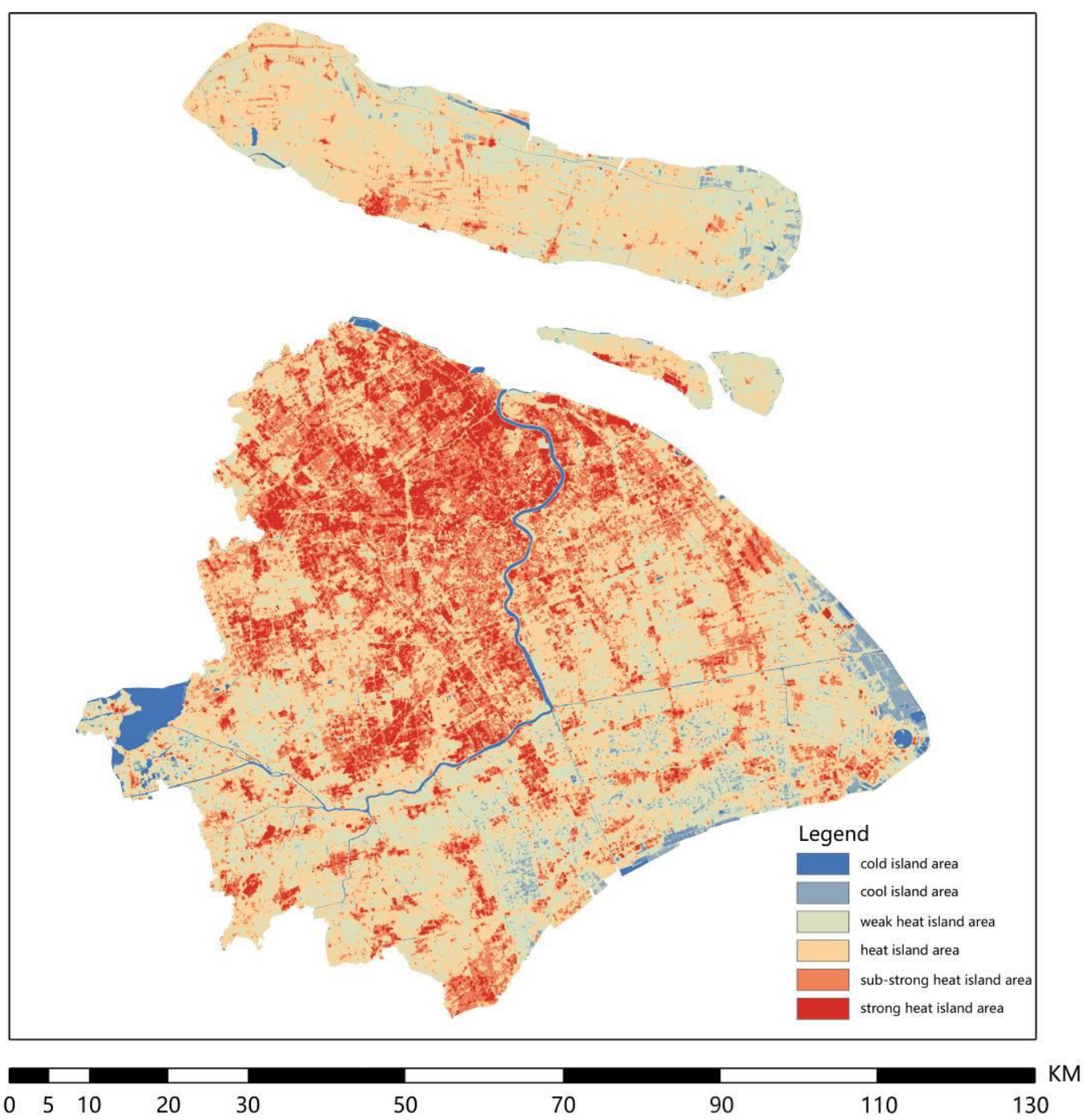

Figure 3 Spatial Distribution Map of Heat Island Effect in Shanghai on July 28, 2016

\subsection{Evolution Analysis of the Urban Heat Island(UHI) Effect in Shanghai}

\subsubsection{Temporal Evolution of UHI}

By comparing the spatial distribution maps of the urban heat island effect of Shanghai in 2000, 2010 and 2016, we can see that the urban heat island effect of Shanghai is stable and obvious in three groups of data in different years, months and meteorological conditions, indicating that the urban heat island effect of Shanghai has been formed and has an aggravating trend, and the spatial distribution of urban heat island has a trend of expansion. Statistical analysis of the temporal variation of urban cold and heat island area in Shanghai (Table 2) shows that the urban heat island effect in Shanghai is increasing from 2000 to 2010, in which the area of the strong heat island area increases the most, accounting for about $55 \%$ of the total increment of the heat island area; from 2010 to 2016, the urban heat island effect in Shanghai eased, the area of the strong heat island area and the heat island area decreased, but the area of the sub-strong heat island area increased by $165.57 \%$. Overall, from 2000 to 2016, the urban heat island area of Shanghai increased by $281.46 \mathrm{~km}^{2}$ and showed a steady increase trend. 
Table 2 Temporal Change of Urban Cold Island and Heat Island Area in Shanghai

\begin{tabular}{|c|c|c|c|c|c|}
\hline \multirow[b]{2}{*}{ Year } & \multicolumn{2}{|c|}{ Urban Cold Island } & \multicolumn{2}{|c|}{ Urban Heat Island } & \multirow{2}{*}{$\begin{array}{c}\text { Urban Cold } \\
\text { VS. Heat } \\
\text { Island }\end{array}$} \\
\hline & Area $\left(\mathrm{km}^{2}\right)$ & Percentage(\%) & Area $\left(\mathrm{km}^{2}\right)$ & Percentage(\%) & \\
\hline 2000 & 2117.64 & 33.40 & 4222.37 & 66.60 & 1: 2 \\
\hline 2010 & 1698.71 & 26.79 & 4641.28 & 73.20 & $3: 8$ \\
\hline 2016 & 1836.16 & 28.96 & 4503.83 & 71.03 & $2: 5$ \\
\hline
\end{tabular}

With the increase of urban heat island area, Qingliang Island area shows a trend of decreasing year by year and accelerating the decline rate, from $278.10 \mathrm{~km}^{2}$ in 2000 to 153.96 $\mathrm{km}^{2}$ in 2016. The reason is that in the process of rapid economic development and urban expansion, construction land replaces a large number of natural vegetation and greening. In addition, while the area of strong heat island and sub-strong heat island expands, the area of cool island decreases synchronously, and some of them are polarized by temperature, which transforms into cold island area. The area of cold island area shows a trend of increasing year by year. From 2000 to 2016, it increased by $86.98 \mathrm{~km}^{2}$ with an average annual growth rate of $14.19 \%$. It is the fastest growth rate of area in all levels of temperature zone. The above changes indicate that the area of extreme temperature zones in Shanghai has grown larger and larger in the past 16 years. Among the six temperature grades, the area of the two extreme grade zones, the strong heat island zone and the cold island zone, has the fastest annual growth rate and an aggravating trend. Relatively speaking, the area of the weak heat Island zone and the cool island zone are gradually declining. However, these two grades are the most livable temperature conditions.

\subsubsection{Spatial Evolution of UHI}

By analysing the spatial distribution maps of urban heat island effect in Shanghai in 2000, 2010 and 2016, it can be seen that the spatial distribution and structure of urban heat island effect in Shanghai have obvious regularity. Generally speaking, the main cold sources regulating urban heat island effect are large areas of water and green space, such as Huangpu River, Dianshan Lake, Dishui Lake, Gulf National Forest Park, Chongming Dongtan Wetland Park and so on. The main heat sources aggravating the heat island effect are industrial, commercial, station, square and high-density residential areas.

In 2000, the spatial distribution of urban heat island in Shanghai was mainly sub-strong heat island area and heat island area, and the proportion of strong heat island area was relatively small, only in traditional industrial and commercial concentrated areas. Overall, in 2000, the urban cold island area of Shanghai was $217.64 \mathrm{~km}^{2}$ and the heat island area was $4222.37 \mathrm{~km}^{2}$, the ratio of which was approximately 1:2. Urban heat islands are concentrated in the central area, while suburban heat islands are few and scattered. The overall spatial structure of heat island is "centralized in the central area and scattered in the suburbs". The inner heat island space in the central area presents a single-center circle-layer distribution. The pattern of scattered heat island in the suburbs basically corresponds to the spatial distribution of new towns and central towns in the suburbs of Shanghai in 2000, including Baoshan Industrial Zone, Jiading New Town, Anting Town, Zhoupu Town and Chuansha Town.

From 2000 to 2010, the urban heat island effect of Shanghai was further aggravated, and the urban heat island showed a trend of expansion. Among them, the area of the strong heat island increased by $230.07 \mathrm{~km}^{2}$, the area of the sub-strong heat island increased by 141.25 $\mathrm{km}^{2}$ and the area of the heat island increased by $47.59 \mathrm{~km}^{2}$. The spatial expansion of the strong heat island area is very significant during 2000-2010. The Shanghai heat island effect 
has initially shown the spatial pattern of "main heat island + corridor + secondary heat island". The central area is the "main heat island" of the heat island effect, and a "hot corridor" is formed along the main urban transportation network, which extends to the suburbs and links several "secondary heat islands". In the central area, "main heat island" mainly distributes in the West Bank Industrial Zone of Huangpu River, Nanjing East RoadNanjing West Road Business District, Xujiahui Business District, etc.; "hot corridor" mainly along Shanghai-Nanjing Expressway and Shanghai-Hangzhou Expressway; "secondary heat island" mainly includes Songjiang, Jiading, Qingpu, Nanqiao and other suburban New cities.

From 2010 to 2016, the area of urban heat island declined considerably, and the urban heat island effect of Shanghai was alleviated. However, the specific analysis shows that part of the heat island region temperature increases, transforms into sub-strong heat island region, and the area of strong heat island region decreases slightly. Overall, since the 12th Five-Year Plan of Shanghai, urban development has focused on ecological environment protection, high-quality healthy urbanization, and the urban heat island effect has been alleviated. Spatially, the mitigation degree of the heat island effect in the central area is more obvious than that in the suburbs. The urban heat island has formed a spatial pattern of "multi-center + two circles + three corridors". The spatial distribution of the urban heat island coincides with the main urban construction areas. By 2016, except for the heat island area formed in 2010, there were local heat island effects in suburban central towns and market towns. To a large extent, the impact of urban construction on the heat island effect is existent and obvious.

\section{Spatial Impact of Urban Construction on Heat Island Effect in Shanghai}

Under the urban scale, the scale, structure and land use types of urban construction in Shanghai have different effects on the intensity and pattern of urban heat island effect. This paper chooses four indicators, including the scale of built buildings, the scale of permanent population, spatial structure and land use types, to analyze their spatial impact on the heat island effect.

\subsection{The Impact of the Scale of Buildings Built on the Heat Island Effect}

The increasing scale of urban buildings affects urban wind speed, direction, energy consumption and heat emission. On the one hand, the existence of buildings changes the properties of the underlying surface of the land, improves the roughness of the underlying surface, and reduces the wind speed of the built area. At the same time, the height of buildings varies affects the urban wind direction. On the other hand, city buildings are endowed with different functions. While operating and maintaining different functions, energy consumption and heat emission are aggravated. For example, air conditioning operation of high-rise office buildings in summer releases more heat.

\subsection{The Impact of Resident Population Size and Density on the Heat Island Effect}

In the process of urbanization, the total resident population size affects the intensity of human activities' impact on the urban environment. The increase of the total population size will increase the energy consumption and heat emission of the city as a whole. The increase 
of population density will increase the heat emission per unit area of land. At the same time, the increase of the size and density of the resident population will bring about the demand for regional transportation and residence, energy consumption and energy consumption. Heat emissions will further increase, aggravating the urban heat island effect.

\subsection{Impact of Land Use Types on Heat Island Effect}

Different types of land have different underlying physical characteristics. The surface albedo is different. The amount of solar radiation absorbed is different, and the land is endowed with different urban functions by man-made, and the way of thermal effect is different, so the surface temperature has different processes. The influence of degree aggravates or slows down the urban heat island effect in varying degrees. Among all kinds of land use types, there are land use which obviously aggravates the heat island effect, such as residential land, industrial and mining storage land, business clothing/public use land, transportation land, etc. There are also land use which significantly slows down the heat island effect and produces local urban cold island, such as cultivated land, woodland, grassland, garden land, water area, etc.

Heat island effect of residential, industrial and mining land

\subsubsection{Heat Island Effect of Residential, Industrial and Mining land}

By classifying urban heat island in Shanghai on July 28, 2016 according to land use types, the underlying surface characteristics and heat island effects of land use types in urban built-up areas, such as residential land, industrial and mining storage land, commercial/public Service land and transportation land, are explored (Table 4). The analysis shows that the heat island effect and influence degree of different land use types in Shanghai are different, showing different surface temperature grades. Generally speaking, the average surface temperature is: industrial and mining warehouse land $>$ residential and commercial land $>$ transportation land.

\subsubsection{Cold Island Effect of Green Space and Water Area}

According to the distribution of urban heat island effect in Shanghai on July 28, 2016, the spatial distribution map of urban cold island in Shanghai was extracted (Figure 4). It can be seen that parks, suburban arable land and water in Shanghai constitute urban cold island, which plays an obvious role in weakening urban heat island effect. 


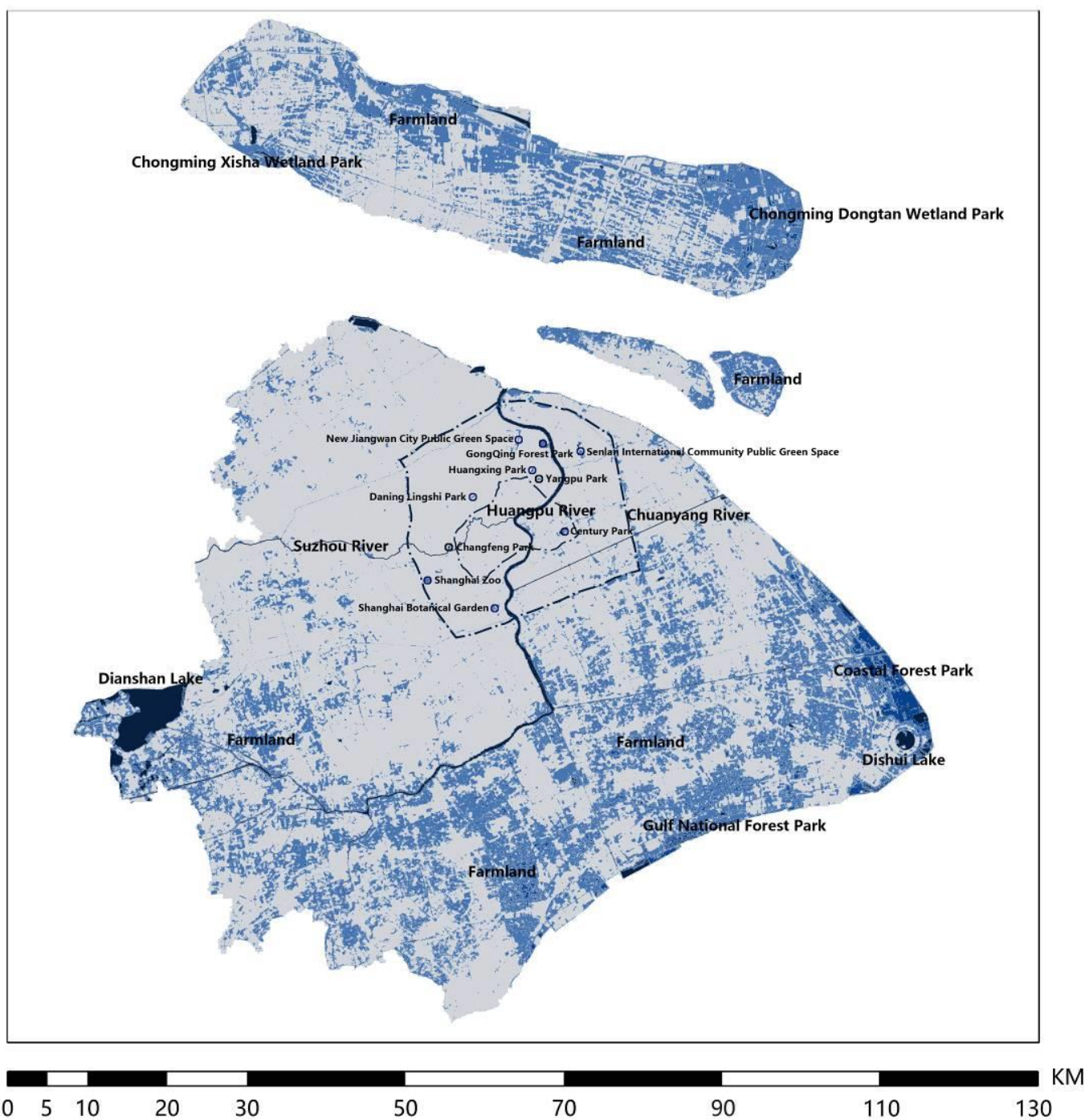

Figure 4 Spatial Distribution Map of Shanghai Urban Cold Island on July 28, 2016

This paper chooses 4 green spaces in Shanghai suburbs and 13 green spaces in the central area as the research objects, carries out "symmetrical" buffer analysis respectively, and counts the information of area, shape and spatial distance to mitigate the heat island effect (Table 3), and studies the spatial impact of green space on urban heat island effect.

Table 3 Statistical Table of Typical Greenbelt Information in Shanghai

\begin{tabular}{|c|c|c|c|c|c|}
\hline \multirow{4}{*}{ Location } & Green Space & $\begin{array}{c}\text { Area } \\
\left(\mathrm{hm}^{2}\right)\end{array}$ & $\begin{array}{c}\text { Block } \\
\text { morphology }\end{array}$ & $\begin{array}{c}\text { Average } \\
\text { Temperature } \\
\left({ }^{\circ} \mathrm{C}\right)\end{array}$ & $\begin{array}{c}\text { Spatial } \\
\text { Mistance of } \\
\text { Mitigating } \\
\text { Heat Island } \\
\text { Effect } \\
(\mathrm{m})\end{array}$ \\
\hline \multirow{3}{*}{ Suburb } & Gulf National Forest Park & 23.99 & Rectangle & 26.32 & $300-500$ \\
\cline { 2 - 5 } & Coastal Forest Park & 8.10 & Rectangle & 25.95 & $100-300$ \\
\cline { 2 - 6 } & $\begin{array}{c}\text { Chongming Dongtan Wetland } \\
\text { Park }\end{array}$ & 181.00 & Rectangle & 25.83 & $1000-1200$ \\
\hline $\begin{array}{c}\text { City } \\
\text { Center }\end{array}$ & $\begin{array}{c}\text { New Jiangwan City Public } \\
\text { Green Space }\end{array}$ & 25.30 & Banding & 27.10 & $300-500$ \\
\hline
\end{tabular}




\begin{tabular}{|c|c|c|c|c|c|}
\hline \multirow{4}{*}{} & $\begin{array}{c}\text { Senlan International } \\
\text { Community Public Green } \\
\text { Space }\end{array}$ & 29.27 & Banding & 26.75 & $500-900$ \\
\cline { 2 - 5 } & GongQing Forest Park & 29.50 & Rectangle & 27.52 & $300-500$ \\
\cline { 2 - 6 } & Huangxing Park & 62.40 & Rectangle & 26.51 & $900-1200$ \\
\cline { 2 - 6 } & Yangpu Park & 22.36 & Rectangle & 27.80 & $100-300$ \\
\cline { 2 - 6 } & Daning Lingshi Park & 68.00 & Irregular & 26.42 & $900-1200$ \\
\cline { 2 - 6 } & Changfeng Park & 36.60 & Irregular & 27.13 & $500-900$ \\
\cline { 2 - 6 } & Century Park & 140.30 & Irregular & 26.32 & $1200-1500$ \\
\cline { 2 - 6 } & Shanghai Zoo & 74.30 & Irregular & 26.47 & $900-1200$ \\
\hline
\end{tabular}

Green space has a significant cooling effect on the surrounding area, and the cooling effect decreases with the increase of distance. Location, area and shape have an impact on the spatial impact distance of Mitigating Heat Island effect.

A.Location impact of green space: The same area of green space, suburban and central green space for mitigating the heat island effect of the spatial distance is different. And when the green space area is small, the cooling effect of suburban coastal green space is lower than that of central green space of the same area; when the green space area is large, the cooling effect of suburban coastal park green space and central park green space of the same area is basically the same.

B.The influence of green space area: On the premise of the same location and shape, the larger the green space area, the lower the average surface temperature of the green space itself, and the larger the spatial impact scope of mitigating the heat island effect in the surrounding area.

C. The influence of green space shape: On the premise of the same location, the larger the ratio of perimeter to area of green space, the more obvious the mitigation of heat island effect.

\section{References}

Ding, Jincai; Zhou, Hongmei (2002) "The Significance of Urban Greening from the Evolution of Shanghai Heat Island", Meteorological, Vol. 2 No. 22-24 (April).

Tan, Jianguo; Zheng, Youfei; Peng,Li; Gu, Songqiang; Shi,Jun (2008) "Effect of Urban Heat Island on Summer High Temperature Heat Wave in Shanghai", Plateau Meteorology,Vol.S1 No.144-149(January).

Zhao, Zongci; Wang, Shaowu; Luo, Yong; Jiang, Ying (2009) "Uncertainty Analysis of Climate Warming in Recent Centuries", Science and Technology,Vol.23 No.41-48(October).

Jiménez-Munoz J C,Sobrino J A(2003) “A Generalized Single-channel Method for Retrieving Land Surface Temperature from Remote Sensing Data",Journal of Geohpysical Research,Vol.108(D22) No.4688-4695.

Qin,Zhihao; Zhang,Minghua;Arnon,Karnieli;et al. (2001) “A Single Window Method for Calculating Surface Temperature Using Landsat TM6 Data", Geography Journal ,Vol.4 No.456-466(April). 
Zhou, Hongmei; Zhou Chenghu; Ge Weiqiang; et al. (2001)"Study on Urban Thermal Field Distribution Based on Remote Sensing and GIS", Geography Journal,Vol.02 No.189197(February).

Zhou, Shuzhen;et al.(1985) Introduction to Urban Climatology, Shanghai,China, East China Teachers' University Press.

Zhou Liang(2006) Landscape Pattern and Heat Island Effect in The Process of Urbanization in Beijing , Beijing,China,Beijing Forestry University.

Peng, Baofa; Shi, Yishao; Wang, Hefeng; Wang, Yali (2013) "The Mechanism of Urban Heat Island Effect and Its Law of Action - Taking Shanghai as an Example", of Geography Journal, Vol.11 No. 1461-1471(November).

Yue Wenze;Xu Lihua (2013) "Thermal Environmental Effects of Typical Urban Waterscape Landscape", Ecology Journal, Vol.06 No.1852-1859(June). 